\title{
Application of a low cost telemedicine link to the diagnosis of neonatal congenital heart defects by remote consultation
} H C Mulholland, F Casey, D Brown, N Corrigan, M Quinn, B McCord, J Rogers,
B G Craig
The rapidly developing field of telemedicine has already found applications in several areas of medicine, including radiology, ${ }^{1-3}$ psychiatry, ${ }^{4}$ dermatology, ${ }^{56}$ and obstetrics. ${ }^{7}$ In particular radiologists have used modern telecommunications systems to transmit still images of standard $x$ rays, computed tomographs, and magnetic resonance imaging scans. Previously reported applications to cardiology have mainly involved ECG transmission. ${ }^{8}$ Most published studies have, however, only shown that particular applications are feasible and the results have not been subjected to qualitative or quantitative analysis. Those projects that have shown a significant clinical benefit are mainly in specialties where imaging and its interpretation are central to the diagnosis or management of the patient. This is particularly true of congenital heart disease, where echocardiography (real time ultrasound imaging) is the primary diagnostic tool. With severe defects, early diagnosis may be vital to allow appropriate management to be instituted within days or even hours after birth. Many such babies are born in the maternity units of district general hospitals remote from immediate access to a consultant paediatric cardiology opinion. The exclusion or confirmation of the diagnosis of major congenital heart disease therefore usually means transfer of the critically ill infant to a regional paediatric cardiology centre for assessment and echocardiography.

Our aim in this study was to determine whether the use of a telemedicine link could assist in the earlier diagnosis of congenital heart disease in an area hospital remote from the paediatric cardiology referral centre. We had three main aims: first, to determine whether echocardiographic images transmitted from a low cost telemedicine system across two integrated services digital network (ISDN) lines would be of adequate quality to confirm or exclude congenital heart disease (ISDN lines are digital telephone lines with the capacity to carry more information at higher speeds than standard telephone lines); second, to determine whether the diagnostic accuracy of the scan performed by the paediatrician can be improved by "live" discussion with the paediatric cardiologist during image acquisition; and third, to assess the impact of the remote consultation on patient management.

\section{Methods}

We established a telemedicine link between Altnagelvin Hospital, Londonderry (a district general hospital) and the regional paediatric
Accepted for publication 25 January 1999
(Heart 1999;82:217-221)

Keywords: congenital heart disease; telemedicine 

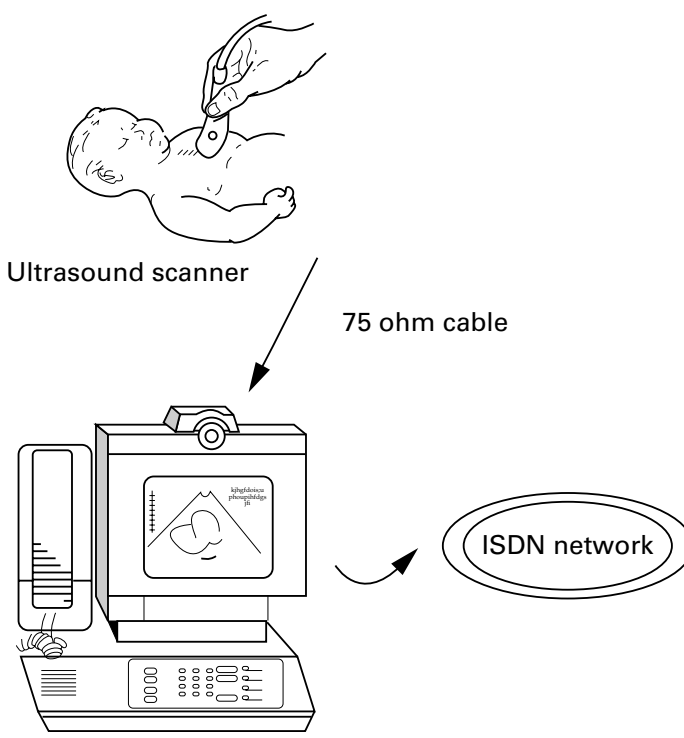

BT VC 7000 Unit

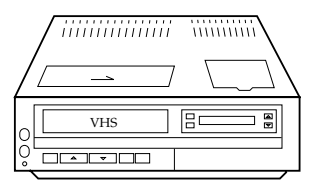

Video recorder

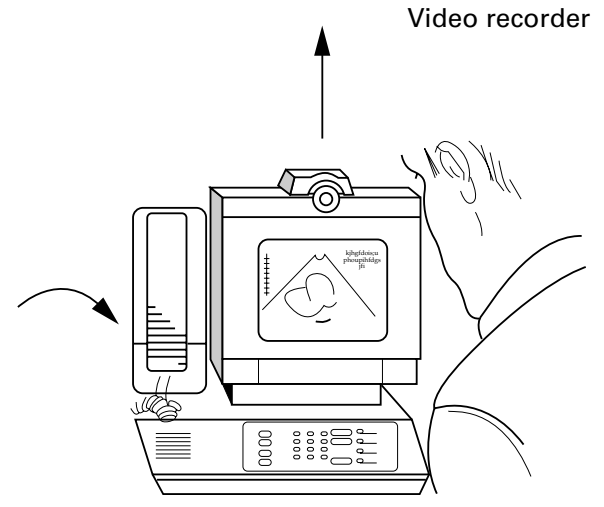

Figure 1 Diagrammatic summary of telemedicine link.

cardiology department at the Royal Belfast Hospital for Sick Children. The distance between the two ends of the link is 75 miles. The link was used to transmit real time echocardiographic images of neonates suspected of having congenital heart disease to the referral centre, with simultaneous video and audio contact for consultation. Echocardiography was performed by a consultant paediatrician and interpreted at the receiving end by a consultant paediatric cardiologist. Each transmitted study was recorded on videotape at the receiving end.

The ultrasound images were acquired in Altnagelvin using an Aloka ultrasound scanner (Aloka UGR38B Tokyo, Japan), the commercially available scanner in routine use on the neonatal unit. The scanner used had pulsed Doppler but not colour Doppler capability. It was connected by cable to a freestanding BT VC7000 video conferencing unit (British Telecommunications, London, UK). The VC7000 is a commercially available Codec designed for business applications. It converts the ultrasound image to a digitised telephone signal which is transmitted across two ISDN lines to the regional paediatric cardiology centre, where a second unit reverses the process so that the paediatric cardiologist can view the images as they are obtained. Figure 1 is a diagrammatic summary of the system.

The feasibility of attempting remote diagnosis using this system was first assessed in a pilot study $^{9}$ on 10 neonates before establishing the study protocol outlined below. An initial scan was performed on each neonate by the paediatrician, who made a tentative diagnosis before seeking consultation with a paediatric cardiologist (scan 1). Having contacted the paediatric cardiologist, echocardiography was then repeated under his guidance using the live telemedicine link (scan 2). All scans performed by the paediatrician were followed by a transmitted scan, including those considered to be normal on scan 1 . Following scan 2, a diagnosis and management plan were agreed between the paediatrician and the paediatric cardiologist. The management options considered were:

1. Urgent transfer to the regional paediatric cardiology unit.

2. Elective transfer to the regional paediatric cardiology unit.

3. Continued management at the district general hospital, with review at the next local paediatric cardiology clinic (performed on a monthly basis by a visiting paediatric cardiologist).

The third step in the process was a direct consultation by the paediatric cardiologist including echocardiography (scan 3), either after referral to the paediatric cardiology unit when this was considered necessary, or at a later outpatient review in the district general hospital. All patients, including those considered to have normal hearts on the transmitted echocardiogram, had an outpatient review and echocardiography. The study protocol is summarised in fig 2 .

\section{Results}

Echocardiograms were transmitted on 63 patients suspected of having congenital heart disease (age range 1 to 42 days). The transmitted images were of sufficient quality for the paediatric cardiologist to give a diagnosis in 61 cases $(97 \%)$. In two cases the transmitted pictures were not of adequate quality to make a reliable diagnosis. The first was a 17 day old infant with ichthyosis and severe desquamation in whom the quality of the transmitted images was poor, partly because of the baby's skin condition, giving a poor "echocardiographic window." In a second patient, considered by the paediatrician to have reduced volume femoral pulses, the paediatric cardiologist 


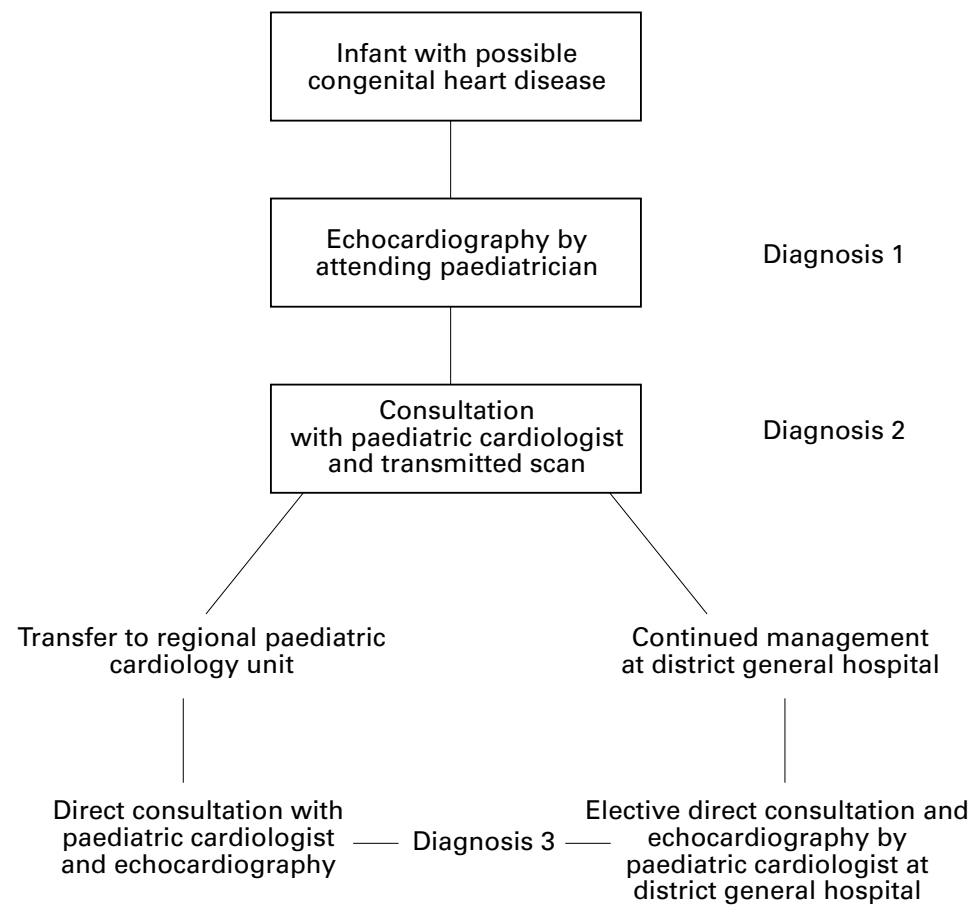

Figure 2 Study protocol.
Table 2 , Summary of diagnoses in 28 patients with "minor" congenital heart disease who continued to be managed at the district general hospital after diagnosis by remote consultation

Small ventricular septal defect

Patent arterial duct

Atrial septal defect plus ventricular septal defect

Ventricular septal defect plus patent arterial duct

diagnosis and management plan were obtained by remote consultation within 24 hours of admission to the district general hospital. After initial measures to stabilise the patient, urgent transfer to the regional cardiology unit was arranged in each case. The clinical presentation and scan results of the patients with major lesions requiring either urgent or elective transfer are summarised in table 1 .

Twenty eight patients (46\%) with more minor congenital heart disease continued to be managed at the district general hospital and were subsequently seen by the visiting paediatric cardiologist at the next clinic. The diagnoses of this group of patients are detailed in table 2 . The need for a potentially hazardous transfer to obtain a diagnosis was therefore avoided in 47 infants $(74 \%)$ - that is, 28 with minor congenital heart disease and 19 with no abnormality.

There were four errors in diagnosis on the transmitted scan (6.7\%). In three patients small muscular ventricular septal defects were not detected. A fourth patient who had a ventricular septal defect detected also had a moderate degree of pulmonary stenosis, which was not diagnosed until the follow up outpatient visit.

Table 1 Summary of clinical presentation and scan results of patients with major congenital heart disease requiring transfer to regional paediatric cardiology unit

\begin{tabular}{|c|c|c|c|c|c|}
\hline Patient & Clinical problems & $\begin{array}{l}\text { Diagnosis } 1 \text {, from scan by } \\
\text { paediatrician }\end{array}$ & $\begin{array}{l}\text { Diagnosis 2, from transmitted } \\
\text { scan }\end{array}$ & Management plan & $\begin{array}{l}\text { Diagnosis 3, after } \\
\text { examination and echo by } \\
\text { cardiologist }\end{array}$ \\
\hline 1 & $\begin{array}{l}\text { Male } 1 \text { day old; cyanotic, } \\
\text { dysmorphic }\end{array}$ & $\begin{array}{l}\text { Complex congenital heart } \\
\text { disease }\end{array}$ & $\begin{array}{l}\text { Single ventricle; } \\
\text { ?hypoplastic left heart }\end{array}$ & $\begin{array}{l}\text { Urgent transfer to } \\
\text { paediatric cardiology unit }\end{array}$ & $\begin{array}{l}\text { Hypoplastic left heart; } \\
\text { interrupted aortic arch }\end{array}$ \\
\hline 2 & Male 6 weeks; CCF & Poor ventricular function & $\begin{array}{l}\text { Poor LV function; ?dilated } \\
\text { cardiomyopathy }\end{array}$ & $\begin{array}{l}\text { Elective transfer after } \\
\text { stabilisation }\end{array}$ & $\begin{array}{l}\text { Poor LV function; ?dilated } \\
\text { cardiomyopathy }\end{array}$ \\
\hline 3 & Female 14 weeks; CCF & $\begin{array}{l}\text { Complex CHD; small LV; } \\
\text { common atrium }\end{array}$ & $\begin{array}{l}\text { Supracardiac total } \\
\text { anomalous pulmonary } \\
\text { venous drainage }\end{array}$ & $\begin{array}{l}\text { Urgent transfer to regional } \\
\text { paediatric cardiology unit }\end{array}$ & $\begin{array}{l}\text { Supracardiac total } \\
\text { anomalous pulmonary } \\
\text { venous drainage }\end{array}$ \\
\hline 4 & $\begin{array}{l}\text { Female twin, } 1 \text { day, born at } \\
25 \text { weeks; cyanosis }\end{array}$ & Complex cyanotic CHD & $\begin{array}{l}\text { Pulmonary valve atresia; } \\
\text { hypoplastic main and } \\
\text { branch PAs; PDA }\end{array}$ & $\begin{array}{l}\text { Elective transfer after } \\
\text { stabilisation with } \\
\text { prostaglandin infusion }\end{array}$ & $\begin{array}{l}\text { Pulmonary valve atresia } \\
\text { hypoplastic main and } \\
\text { branch PAs; PDA }\end{array}$ \\
\hline 5 & $\begin{array}{l}\text { Female, } 7 \text { weeks; CCF, } \\
\text { cyanosis }\end{array}$ & $\begin{array}{l}\text { Transposition of great } \\
\text { arteries and VSD }\end{array}$ & $\begin{array}{l}\text { Transposition of great } \\
\text { arteries; VSD; ASD; PDA }\end{array}$ & $\begin{array}{l}\text { Urgent transfer to regional } \\
\text { paediatric cardiology unit }\end{array}$ & $\begin{array}{l}\text { Transposition of great } \\
\text { arteries; VSD; ASD; PDA }\end{array}$ \\
\hline 6 & Male, 1 week; CCF & VSD; ?coarctation of aorta & $\begin{array}{l}\text { Moderate sized VSD; } \\
\text { coarctation of aorta }\end{array}$ & $\begin{array}{l}\text { Urgent transfer to regional } \\
\text { paediatric cardiology unit }\end{array}$ & $\begin{array}{l}\text { Moderate sized VSD; } \\
\text { coarctation of aorta }\end{array}$ \\
\hline 7 & $\begin{array}{l}\text { Male, } 1 \text { day; tachycardia; } \\
\text { CCF }\end{array}$ & ?Atrial flutter & $\begin{array}{l}\text { Atrial flutter; moderate TR; } \\
\text { reduced ventricular } \\
\text { function }\end{array}$ & $\begin{array}{l}\text { Urgent transfer to regional } \\
\text { paediatric cardiology unit }\end{array}$ & $\begin{array}{l}\text { Atrial flutter; moderate TR; } \\
\text { reduced ventricular } \\
\text { function }\end{array}$ \\
\hline 8 & Male, 2 days; cyanosis & Complex CHD & $\begin{array}{l}\text { Double outlet right } \\
\text { ventricle; anterior aorta; } \\
\text { pulmonary stenosis }\end{array}$ & $\begin{array}{l}\text { Urgent transfer to regional } \\
\text { paediatric cardiology unit }\end{array}$ & $\begin{array}{l}\text { Double outlet right } \\
\text { ventricle; anterior aorta; } \\
\text { pulmonary stenosis }\end{array}$ \\
\hline 9 & Female, 2 days; CCF & Complex CHD & $\begin{array}{l}\text { Hypoplastic left heart } \\
\text { syndrome }\end{array}$ & $\begin{array}{l}\text { Urgent transfer to regional } \\
\text { paediatric cardiology unit }\end{array}$ & $\begin{array}{l}\text { Hypoplastic Left heart } \\
\text { syndrome }\end{array}$ \\
\hline 10 & Female, 2 days; CCF & Complex CHD & $\begin{array}{l}\text { Hypoplastic left heart } \\
\text { syndrome }\end{array}$ & $\begin{array}{l}\text { Urgent transfer to regional } \\
\text { paediatric cardiology unit }\end{array}$ & $\begin{array}{l}\text { Hypoplastic left heart } \\
\text { syndrome }\end{array}$ \\
\hline 11 & Male, 1 day; cyanosis & $\begin{array}{l}\text { ?Transposition of great } \\
\text { arteries }\end{array}$ & $\begin{array}{l}\text { Transposition of great } \\
\text { arteries; intact ventricular } \\
\text { septum; PFO }\end{array}$ & $\begin{array}{l}\text { Urgent transfer to regional } \\
\text { paediatric cardiology unit }\end{array}$ & $\begin{array}{l}\text { Transposition of great } \\
\text { arteries; intact ventricular } \\
\text { septum; PFO }\end{array}$ \\
\hline 12 & Male, 10 days; CCF & $\begin{array}{l}\text { Perimembranous VSD; } \\
\text { ?aortic override }\end{array}$ & $\begin{array}{l}\text { Large outlet VSD; ?small } \\
\text { ASD }\end{array}$ & $\begin{array}{l}\text { Elective transfer to regional } \\
\text { paediatric cardiology unit }\end{array}$ & Large outlet VSD; PFO \\
\hline 13 & $\begin{array}{l}\text { Male, } 4 \text { days; poor feeding, } \\
\text { systolic murmur }\end{array}$ & Complex cyanotic CHD & Tetralogy of Fallot & $\begin{array}{l}\text { Elective transfer to regional } \\
\text { paediatric cardiology unit }\end{array}$ & Tetralogy of Fallot \\
\hline 14 & Female, 5 days; CCF & ASD & ASD; large VSD; PDA & $\begin{array}{l}\text { Elective transfer to regional } \\
\text { paediatric cardiology unit }\end{array}$ & ASD; large VSD; PDA \\
\hline
\end{tabular}

ASD, atrial septal defect; CCF, congestive cardiac failure; CHD, congenital heart disease; LV, left ventricle; PA, pulmonary artery; PDA, patent arterial duct; PFO, patent foramen ovale; TR, tricuspid regurgitation; VSD, ventricular septal defect. 
The initial scan performed by the paediatrician (scan 1) was accurate in $66 \%$ of cases. Accuracy of diagnosis was improved to $91 \%$ on the transmitted scan (scan 2), illustrating the benefit of the telemedicine link (sensitivity $90.47 \%$, specificity 97\%). The diagnostic errors on scan 1 included both false positives and false negatives. The lesions not detected on scan 1 included five patients with ventricular septal defects and two patients with pulmonary valve stenosis. In seven cases where there was a major abnormality the diagnosis on scan 1 was "complex congenital heart disease." In these cases the diagnosis was only defined on scan 2 and an appropriate management plan instituted at that point. There were three false positive diagnoses of coarctation and one of atrial septal defect on scan 1 .

\section{Discussion}

Our study, as far as we are aware, is the first report of the use of a telemedicine link using ISDN lines to diagnose congenital heart disease from transmitted real time echocardiographic images obtained with the assistance of "live" guidance by the paediatric cardiologist. Transmission of high quality live fetal ultrasound images has been successfully achieved by Fisk et al using ISDN lines. ${ }^{7}$ There are two previous reports of remote echocardiographic diagnosis of congenital heart disease. Sobczyk et al reported successful transmission of previously recorded echocardiographic images using a transtelephonic link, ${ }^{10}$ while Finlay et al also reported transmission of echocardiographic images, initially using a more expensive microwave radio system and more recently a broadband video circuit. ${ }^{11}{ }^{12}$

The system we used consists of currently available, relatively inexpensive video conferencing equipment, with the signal transmitted over ISDN lines at a bandwidth of 128 kbits/second. The rental cost of the total system is approximately $£ 3000$ (US $\$ 4800$ ) per annum. The cost of transmitting each scan is similar to that of two standard telephone calls of approximately 30 minutes' duration. The ultrasound images were obtained on a commercially available scanner, similar to that available in almost all neonatal units.

In contrast to other published studies we have achieved $100 \%$ patient follow up to verify the accuracy of remote diagnosis by this method. Our findings from this study suggest that, for babies suspected of having congenital heart disease, ultrasound images of diagnostic quality can be obtained and transmitted using a low cost system. It is obviously of some concern that in four cases abnormalities were missed on the transmitted scan. While none was life threatening, nor a reason to transfer the patient to a regional centre, it would be desirable to identify all lesions if possible. The detection of small ventricular or atrial septal defects is beyond the resolution achieved on the transmitted image using our present system, particularly without the use of colour Doppler. The detection of such defects was not, however, the purpose of this study. Elective clinical consultation at a later time by a paedi- atric cardiologist for those patients considered to have minor lesions or normal hearts on the transmitted scan is an important part of the process. Remote echocardiography should not be seen as replacement of this consultation.

In terms of life or death, the defects which must be diagnosed are those causing cyanotic heart disease, major obstructive lesions of the left or right heart, and total anomalous pulmonary venous drainage (TAPVD). The latter is likely to be the most difficult diagnosis to make, though it is obviously of critical importance, particularly in those babies where the differential diagnosis is persistent fetal circulation. In the series to date there has been only one patient with TAPVD. The correct diagnosis of supracardiac TAPVD was made with the transmitted scan, on which the pulmonary venous confluence and the ascending vein could be seen. In this case the initial diagnosis by the paediatrician was hypoplastic left heart. The exclusion of a diagnosis of TAPVD requires a combination of imaging the pulmonary veins entering the left atrium and the demonstration of normal Doppler flow from the pulmonary veins into the left atrium. In the cases to date where a diagnosis of persistent fetal circulation was being considered we have been able to satisfy ourselves that pulmonary venous drainage was normal.

In this preliminary work, patients with major defects were identified and appropriate management instituted earlier than would have been the case had the remote consultation not occurred. The potential benefits of the link have to be balanced against the possible risk of "false reassurance" that the heart is normal in cases when in fact a major defect is present. In the study to date this has not occurred. We hope that with increasing numbers more precise information on the sensitivity of this system will be obtained.

The accuracy of the diagnosis is dependent on the quality of the images obtained by the person transmitting the scan. In this study, consultant paediatricians, none of whom had any formal training in performing echocardiography, obtained all the ultrasound images. As paediatricians will vary widely in their experience in performing ultrasound studies, we recommend that those intending to become involved in performing scans using the telemedicine link should attend a basic paediatric echocardiography course. We hoped that one of the potential benefits of this link would be an educational one, in that the skills of the paediatrician might be improved by performing scans under the guidance of a paediatric cardiologist. It became apparent that as the study progressed those performing the scans were obtaining the appropriate views with less difficulty.

The second major variable influencing the accuracy of the diagnosis is the degree of loss of image quality during transmission. Using our present system with ISDN2 giving a bandwidth of $128 \mathrm{kbits} / \mathrm{second}$ there is undoubtedly some loss of image quality, although we have as yet not been able to quantify this. The transmitted image is of sufficient quality to allow diagnosis 
or exclusion of major defects, but more minor defects cannot be excluded. In trying to reduce the "image quality loss" factor there has to be a balance between what is desirable from the clinical perspective and what is cost and time effective. We have now begun to study the diagnostic accuracy achievable with ISDN4 and ISDN6. The improved image quality of the higher bandwidth should increase the yield of minor lesions such as small ventricular septal defects but it remains to be seen whether there is a difference in diagnostic accuracy for major lesions.

The effect on patient management was significant. All those with severe congenital heart disease received an early and accurate diagnosis. In $74 \%$ of all patients the need to transfer babies to obtain a diagnosis was avoided. This is of particular benefit in very ill infants-for example, those with persistent fetal circulation, avoiding the need for a potentially dangerous transfer. As well as being of clinical benefit this method of consultation is cost effective, with 47 ambulance transfers avoided. The return ambulance trip from Altnagelvin Hospital to the Royal Belfast Hospital for Sick Children takes a minimum of four hours. The patient being transferred is usually accompanied by a nurse and a doctor of specialist registrar grade. We estimate the cost of each transfer, at current tariffs, to be $£ 300$ (US\$480). The savings to the referring hospital in this two year study period are therefore $£ 14100$ (US\$22 560) minus the cost of equipment rental, and telephone charges, which was approximately $£ 6500$ (US $\$ 10400$ ).

Our early experience with live transmission of echocardiograhic images leads us to believe that this technique has great potential in assisting paediatricians remote from access to a paediatric cardiology centre in making an early and accurate diagnosis of neonatal congenital heart disease. We believe that this is one application of telemedicine for which we have been able to demonstrate both clinical efficacy and financial viability. With increasing patient numbers, appropriate teaching of the personnel involved, and further refinements of the transmitting system, we hope to evaluate and expand the application of this technique further. It is not appropriate or necessary to apply this technology to the assessment of the child where an urgent clinical decision does not need to be made - for example, the evaluation of a murmur. Telemedicine links should be used to diagnose or exclude significant neonatal congenital heart disease. Over the next few years paediatric cardiologists are likely to find that providing remote diagnosis in this way could become an increasingly important part of their workload.

We thank Hearbeat for financial support to fund this project. We are grateful also to $\mathrm{Mr}$ Fred McCullough for technical assistance.

1 Wright R, Loughrey C. Teleradiology. BMF 1995;310:1392-

2 Barnitzky S, Rosenthal SJ, Siegel EL, et al. Teleradiology: assessment. Radiology 1990;177:11-17.

3 Binkhuysen F, Ottes FP, Valk J, et al. Remote expert consulBinkhuysen F, Ottes FP, Valk J, et al. Remote expert consul-
tation for MRI procedures by means of teleradiology. Eur $\mathcal{F}$

Radiol 1995;192:147-50.
4 Brown FW. A survey of telepsychiatry in the USA. 7 Telemed Brown FW. A survey of tele
Telecare 1995;1:19-21.

5 Oakley AMM, Astwood DR, Loane M, et al. Diagnostic accuracy of teledermatology: results of a preliminary study in New Zealand. NZ Med f 1997;110:51-3.

6 Loane MA, Gore HE, Bloomer SE, et al. Preliminary results from the Northern Ireland arms of the UK multicentre teledermatology trial; is clinical management by real-time teledermatology possible? f Telemed Telecare 1998;4(suppl 1):3-5.

7 Fisk NM, Bower S, Sesulveda W, et al. Fetal telemedicine: interactive transfer of real-time ultrasound and video via ISDN for remote consultation. $\mathcal{F}$ Telemed Telecare 1995;1: $38-44$.

8 Ong K, Chia P, Ng WL, et al. A telemedicine system for high-quality transmission of paper electrocardiographic righ-quality transmission of paper electr.

9 Casey F, Brown D, Craig BG, et al. Diagnosis of neonatal Congenital heart defects by remote consultation using a lowcongenital heart defects by remote consultation using a

10 Sobczyk WL, Solinger RE, Rees AH, et al. Transtelephonic echocardiography: successful use in a tertiary pediatric referral centre. $\mathcal{F}$ Pediatr 1993;122:S84-8

11 Finlay JP, Human DG, Nanton MA, et al. Echocardiography by telephone: evaluation of pediatric heart disease at a distance. Am $\mathcal{F}$ Cardiol 1989;63:1475-7.

12 Finlay JP, Sharrat GP, Nanton MA, et al. Echocardiography by telemedicine-nine years experience. $\mathcal{F}$ Telemed Telecare 1997;3:200-4. 